\title{
Un nouveau transactivateur des gènes du CMH-Il muté dans une forme d'immunodéficience héréditaire
}

Les molécules du complexe majeur d'histocompatibilité de classe II (CMH-II) jouent un rôle essentiel dans la réponse immunitaire en présentant les antigènes aux lymphocytes $\mathrm{T}$. Leur expression est strictement contrôlée et normalement restreinte aux cellules présentatrices de l'antigène. Elle peut être modulée par un grand nombre d'effecteurs, dont l'interféron $\gamma$ [1]. L'absence de molécules de classe II à la surface des cellules est caractéristique d'une forme d'immunodéficience primaire, déficience en CMH-II ou syndrome des lymphocytes dénudés (bare lymphocyte syndrome, BLS). Les seules formes de thérapie de cette immunodéficience sévère sont l'isolement des patients de toute source infectieuse et la greffe de moelle osseuse. Le syndrome résulte de l'abolition de la transcription de l'ensemble des gènes du complexe. Du fait que sa ségrégation est indépendante du CMH-II, ses causes sont à rechercher parmi les gènes trans-régulateurs de celui-ci [ 2 , 3]. L'expression des gènes du CMH-II est sous la dépendance de séquences conservées localisées dans les promoteurs de ces gènes et sur lesquelles viennent se fixer de nombreuses protéines (figure 1A), mais le rôle précis de chacune d'elles n'est pas encore élucidé [4]. L'étude du syndrome des lymphocytes dénudés, une maladie de la régulation transcriptionnelle est donc essentielle.

Parmi les malades atteints de cette affection on distingue au moins trois groupes de complémentation A, B et $\mathrm{C}$, mis en évidence par fusion cellulaire. Aucune différence n'est observée par les techniques usuelles d'analyse des promoteurs des gènes entre des groupe de complémentation A et d'individus normaux (jootprint in vivo et in vitro, retard de migration sur gel). Dans les groupes B et C, les complexes protéiques formés sur les promoteurs sont altérés in vivo, en raison de l'absence de la fixation, sur la boîte $\mathrm{X}$ des promoteurs, du complexe appelé RFX (regulatory factor X) ([5] figure $1 B$ ). L'absence de la fixation de RFX dans les groupes de complémentation $\mathrm{B}$ et $\mathrm{C}$ est à l'origine de la maladie.

Le gène impliqué dans le groupe $\mathrm{A}$ a été cloné par une approche de complémentation génétique de cellules mutantes par une banque d'expression d'ADNc de cellules normales (m/s $n^{\circ} 3$, vol. 10, p. 360) [6]. Ce gène, dénommé C:IITA (CMH-II transactivator), est nécessaire pour l'expression constitutive des gènes du CMH-II et représente également un intermédiaire obligatoire pour leur induction par l'interféron $\gamma$ [7]. Son mode d'action précis n'est pas encore élucidé. Il code pour une protéine de $135 \mathrm{kDa}$ qui ne se lie pas directement aux séquences promotrices des gènes du CMH-II. La protéine CIITA pourrait être un coactivateur de la transcription en se liant indirectement aux promoteurs des gènes (figure $1 B$ ).

Le clonage des gènes impliqués dans les groupes B et C a été entrepris par deux approches parallèles. La première approche a consisté à purifier le complexe RFX [8]. L'ajout de RFX purifié à des extraits nucléaires de cellules de malades des groupes B et C suffit à corriger in vitro le défaut de transcription du CMH-II. On démontre ainsi directement que RFX est effectivement le complexe altéré dans les groupes de complémentation B et C. La fraction purifiée contient cinq polypeptides de tailles respectives : $75,62,56,45$ et $36 \mathrm{kDa}$, mais on ne connaît pas le rôle de chacun de ces polypeptides dans le complexe RFX. Par des expériences de couplage covalent de RFX à l'ADN, on a démontré que le polypeptide de $36 \mathrm{kDa}$ est un constituant de RFX et lie l'ADN [8]. (irâce à une approche de clonage par complémentation du groupe C identique à celle employée pour le groupe $A$, le gène codant pour la sous-unité de $75 \mathrm{kDa}$ a été cloné [9]. Ce gène appelé $R F X 5$ code pour une protéine appartenant à une nouvelle famille de facteurs de transcription qui possèdent de très fortes analogies de séquence dans leur domaine de liaison à l'ADN [10]. Comme attendu, le gène $R F X 5$ complémente spécifiquement le groupe $\mathrm{C}$ des cellules de patients atteints de syndrome des lymphocytes dénudés et est muté chez ces malades. La question du rôle de RFX5 dans le complexe RFX a été abordée par ciblage à l'aide de l'épitope HA du virus influenza. $R F X 5$, fusionné à la séquence codant pour cet épitope, est transfecté dans des cellules de malades et ces dernières sélectionnées sur la présence de molécules du CMH-II à leur surface. Avec des extraits nucléaires de ces cellules et à l'aide d'anticorps dirigés contre l'épitope $\mathrm{HA}$, on a pu clairement démontrer que la protéine RFX5 est présente dans le complexe RFX. Enfin, le microséquençage de la protéine de $75 \mathrm{kDa}$ purifiée a permis de démontrer l'identité entre cette sous-unité et RFX5 [9].

En conclusion, les gènes impliqués dans les groupes de complémentation A et C ont été clonés. Le premier code pour un transactivateur essentiel à l'expression constitutive et induc- 


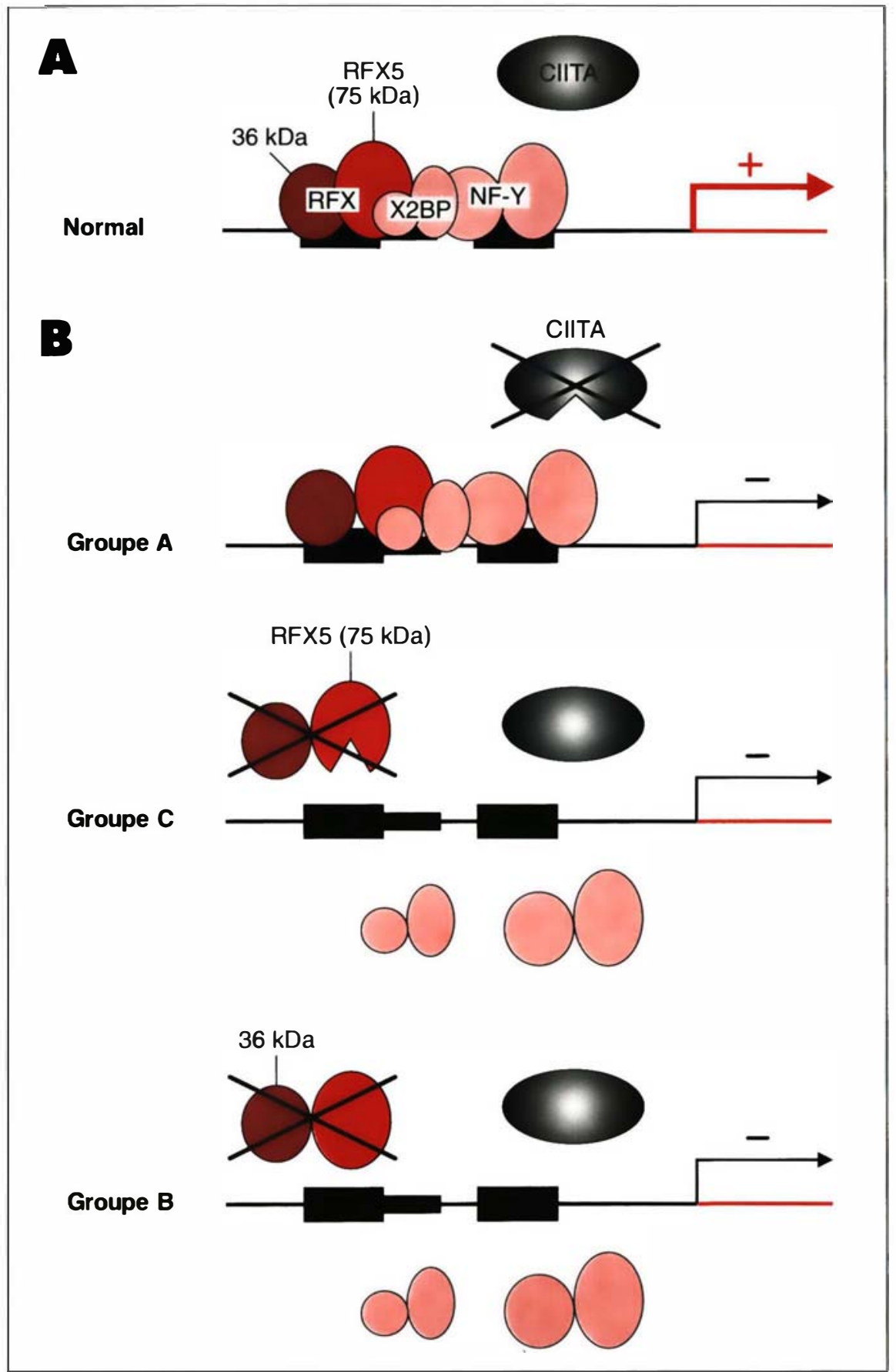

Figure 1. Représentation schématique des défauts moléculaires impliqués dans les trois groupes de complémentation du syndrome des lymphocytes dénudés. A. État d'occupation d'un promoteur dit normal. B. Le transactivateur CIITA est muté chez les patients du groupe $A$. II ne se lie pas directement à I'ADN. Le complexe RFX est absent chez les patients des groupes $B$ et $C$. RFX5, la sous-unité de 75 kDa de RFX est mutée dans le groupe C. La nature de la mutation impliquée dans le groupe $B$ n'est pas élucidée mais pourrait affecter la sous-unité de $36 \mathrm{kDa}$ de RFX ou une protéine non encore identifiée nécessaire à la liaison de RFX à l'ADN. En absence de RFX, les autres complexes tels que X2BP et NF-Y ne peuvent, in vivo, se lier au promoteur.

$\mathrm{m} / \mathrm{s} n^{\circ} 9$, vol. 11 . septembre 95 tible des gènes du CMH-II mais qui ne se lie pas au promoteur. Le deuxième code pour l'une des sousunités du complexe RFX (figure 1B). Il est tentant de spéculer que le gène impliqué dans le troisième groupe de complémentation (B) pourrait être celui qui code pour la sous-unité de 36 kDa de RFX, bien que l'on ne puisse exclure que ce soit un gène qui modifie RFX en lui conférant une activité de liaison à l'ADN (figure 1B). L'élucidation du syndrome des lymphocytes dénudés a donc amélioré notre compréhension des mécanismes de la régulation de la réponse immunitaire. La découverte de ces nouveaux transactivateurs ouvre des voies thérapeutiques nouvelles.

B.D. B.M.

1. (Blimcher L.H, Kara (.J. Sequences and factors a guide to MHC class II transcription. Annu Re Immunol $1992 ; 10: 13-49$.

2. De Préval C., Lisowska-Grospierre B, Loche M (iriscelli C., Mach B. A trans-acting class II regulatory gene unlinked to the MHC: controls expression of HL $\Lambda$ class Il genes. Nature $1985: 318: 291-3$. 3. Lisowska-(irospierre B, (iriscelli (.) Anomalie héréditaire de l'expression des molécules HLA de classe II. médecine/sciences $1987 ; 3: 19-26$.

4. Reith W, Steimle V, Mach B. Molecular defects in the bare lymphocyte syndrome and the regulation of MHC: class II genes. Immunol Today 1995 ; sous presse.

5. Reith W, Satola S, Herrero-Sanchez (., Amaldi 1. Lisowska-(Grospierre B, Griscelli (;, Hadam MR, Mach B. Congenital immunodeliciency with a regulatory defect in $\mathrm{MHC}$; class II gene expression lacks a specific HLA-DR promoter binding protein RFX. Cell $1988 ; 53: 897-906$.

6. Steimle V, Otten L, Zufferey M, Mach B. Com plementation cloning of an MHC-class II transactivator mutated in the MH(i-class II deficiency (bare lymphocyte syndrome). (ell 1993; 75: 135 46.

7. Steimle V. Siegrist C, Mottet A, I isowska-Grospierre B, Mach B. Regulation of MHC-class II expression by interferon $\gamma$ mediated by the transactivator gene CIITA. Srience $1994 ; 265: 106-9$. 8. Durand B, Kobr M, Reith W, Mach B. Functional complementation of an MHC class II regulatory mutant by the purified $\mathrm{X}$ box binding protein RFX. Mol (ell Biol 1994: 14 : 6839-47.

9. Steimle V, Durand B. Barras E. Zufferey M Hadam M, Mach B, Reith W. A novel DNA-binding regulatory factor is mutated in primary MH(-class II deficiency (bare lymphocyte syndrome). (ienes Inn 1995; 9 : 1021-32.

10. Reith W, Ucla (;, Barras E, ( ;aud A, Durand B Herrero-Sanchez (; Kobr M, Mach B. RFXI, a transactivator of the hepatitis $B$ virus enhancer 1 belongs to a novel fanily of homodimeric anc heterodimeric transcription factors. Mol Ciell Biol $1994 ; 14: 12: 30-44$. 